\title{
AN ANALYSIS OF THE RELATIONSHIP BETWEEN NET PROFIT AND OWNER'S EQUITY OF SELECTED CEMENT COMPANIES IN INDIA
}

\author{
*Mr. Nagesha H G
}

**Dr. T P Renuka Murthy

\begin{abstract}
Capital structure decision is the major one since the profitability of a firm is directly affected by such decision. The successful selection and use of capital is one of the key elements of the firms'financial strategy. Hence, proper care and attention need to be given while determining equity in capital structure decision. The purpose of this study is to investigate the relationship between net profit and owner's equity of 10 listed Indian cement companies over the past 5 years period from 2011 to 2016.The data has been analyzed through using descriptive statistics and correlation analysis to find out the association between the variables. Results of the analysis show that there is a positive as well as negative association between net profit and owner's among them.
\end{abstract}

Keywords: Capital Structure, Owner's Equity, Profit.

\section{Introduction}

The relationship between net income and owner's equity is through retained earnings, which is a balance sheet account that accumulates net income. Proper and efficient capital structure decision has an advantage of profit and wealth maximization and minimization of cost of capital. Many factures influence on capital structure of firm. They are collateral value of assets, non debt tax shields, growth, uniqueness, industry classification, size of the firm, volatility, profitability and so on. Owner's equity is the difference between the company's assets and liabilities. It is the owner's share of the proceeds if you were to liquidate the company today.

Owner's equity is the sum of the owner's contributions to his company and retained earnings, minus cash withdrawals. The corresponding term for corporations is "stockholders' equity," which is the sum of the proceeds from issuing stock and retained earnings. Therefore, owner's equity rises when a company generates a profit and retains part of it after paying dividends. Losses lead to lower owner's equity or even negative owner's equity. The owner could put in additional cash to continue operations, sell off surplus assets to
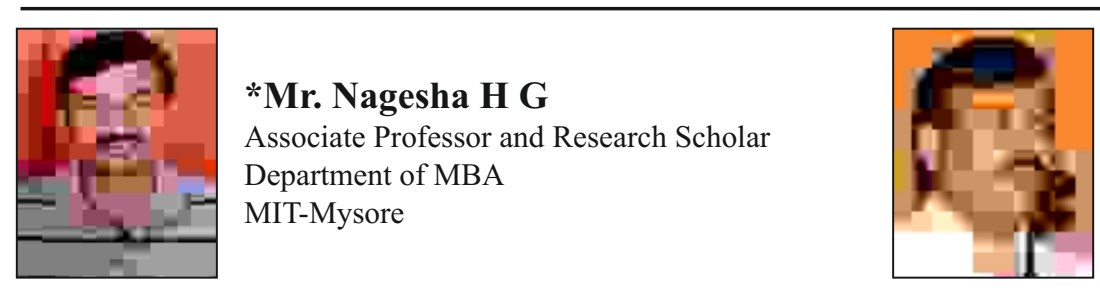

**Dr. T P Renuka Murthy

Professor and Course Coordinator Department of MBA, VTU Post Graduate Centre VTU RO, Mysore 
raise cash or liquidate all assets and shut down the company. The owner's equity is usually a company's book value. The market value could be higher or lower than this book value. The market value is higher when investors are optimistic about a company's prospects for growing revenues and net income. The market value is lower when weak economic and industry fundamentals lead to expectations of flat or lower net income.

The more money a business takes in, the more money its owners are likely to make. A company's net income therefore plays a significant role in determining owner's equity.

How net income affects owner's equity?

Net income contributes to a company's assets and can therefore affect the book value, or owner's equity. When a company generates a profit and retains a portion of that profit after subtracting all of its costs, the owner's equity generally rises . On the flip side, if a company generates a profit but its costs of doing business exceed that profit, then the owner's equity generally decreases.

However, net income is only one factor that can affect owner's equity in a company. Owner's equity can also increase if the owner of a business invests more money into the business. Similarly, it can decrease if the owner takes money out of the business.

In a typical corporation, stockholders' equity has two main elements: paid-in capital and retained earnings. Paid-in capital is simply the money the company has collected from selling stock. It's what stockholders have "paid in" to the company. Retained earnings are all the profits that the company has reinvested over the years. ("Earnings" is just another name for profits). Most corporations aren't constantly selling stock to the public, so it's common for the total amount of paid-in capital to go unchanged over long periods. But retained earnings are regularly in flux, because they're directly affected by net income.

Optimal capital structure which maximizes the shareholders' wealth with best combination of debt and equity mix by minimizing the firms cost of capital to increase the level of activity in the company. They were to raise the debt amount, but it may create the financial risk in the organization. Capital structure decision is crucial for any business organization. The decision is important because of the need to maximize returns to various organizational constituencies and also because of the impact such a decision has an organizational ability to deal with its competitive environment. Optimal capital structure choice can be influenced by factors such as growth, cash flow, size, etc... in attaining an optimal capital structure.

\section{Objective of the study}

There are not many studies on the relationship between net profit and owner's equity.

The study aims to investigate the comparatively under-researched relationship between net profit and owner's equity in an emerging market.

\section{Research Methodology}

The study includes only secondary sources of information i.e., from financial reports of cement company through moneycontrol.com. Based on market capitalization top 10 listed companies were selected in BSE and NSE Indian stock markets. Here the study uses convenience sampling. Because out of 27 companies, only top 10 companies financial reports are available for 5 
years. In order to test the relationshipKarlPearson's correlation is used, Companies are:Ultra tech cement ,Shree cement, Ambuja cement, ACC, Ramco Cement, prism cement, J.K. Cement, J.K. Laxmi Cement, Orient cement and OCL India.

\section{Data Analysis and Findings}

Correlation (r) is used to see the relationship between net profit and owner's equity.

$\mathrm{X}$ is owners' equity and $\mathrm{Y}$ is net profit

\section{SUMMARY OF CORRELATION COMPUTATION TABLE}

\begin{tabular}{|c|c|c|c|}
\hline Company Name & $\begin{array}{c}\text { Average owners' } \\
\text { Equity }\end{array}$ & Average Net Profit & Correlation (r) \\
\hline $\begin{array}{c}\text { Ultra Tech } \\
\text { Cement }\end{array}$ & 2287.148 & 16957.18 & -0.70769 \\
\hline Shree Cement & 658.116 & 5057.508 & -0.38937 \\
\hline Ambuja Cement & 1224.882 & 9353.98 & -0.24398 \\
\hline ACC & 1146.124 & 7815.712 & -0.85001 \\
\hline Ramco Cement & 345.414 & 2528.208 & -0.362106 \\
\hline Prism & -29.616 & 1050.032 & 0.25926 \\
\hline J K Cement & 154.838 & 1578.13 & -0.11137 \\
\hline J K Laxmi & & & -0.49247 \\
\hline Cement & 95.88 & 1280.47 & \\
\hline Orient Cement & 103.728 & 715.266 & -0.683609 \\
\hline OCL India & 127.212 & 1130.59 & 0.859036 \\
\hline
\end{tabular}

The above table says that in ultra tech cement and OCL India there is a positive relationship between owner's equity and net profit and restof 8 companies are negatively correlated.

\section{Summary and Conclusion}

This study investigated the interrelation between net profit and owner's equity using correlation. Firms selected for the study had to have maintained their identity and reported their annual accounts without any significant gaps for the financial years 2011-12 to 2015-16. There were 10 cement firms selected as a sample for this study. Increase in equity capital will create less riskiness in cement companies. This study suggests that there is a correlation between net profit and equity capital. But the level of correlation is very less in an average of Indian cement industry. Hence fixation of owner's equity plays a vital role in capital structure decision for making profit.

\section{References}

T. Velnampy and J AloyNiresh, the relationship between capital structure and profitability, Global Journal of Mangement and Business Research, Vol 12, Issue 13, 2012

Basil Al-Najjar and Peter Taylor, the relationship between capital structure and ownership structure, Emerald Journal,Mangerial Finance, Vol. 34, No.12, 2008

Marc Jegers, the sustainable growth rate of Non Profit Organizations: The Effect of Efficiency, Profitability and Capital structure, Financial Accounting and Management, 19(4), Nov.2003.

Roden, Dianne M, Lewellen and Wilburg, Corporate Capital Structure DecisionEvidence from Leveraged Buyout, Journal of the Financial Management Association, Vol 24, Issue 2.1992.

Chadha, Saurabh, Sharama and Anil K, Capital Structure and Firm Performance: Empirical evidence from India, Business Source Elite, Vol 19, Issue 4

FitimDeari, Media Deari: The Determinants of

Capital Structure: Evidence from Macedonian Listed and Unlisted Companies

RavinderVinayak and Ravi Kumar Gupta: Determinants of Capital Structure of Corporate Giants in India, The Indian journal of commerce, Vol. 55, No.4, Oct-Dec 2002.

A. M. Goyal: Impact of Capital Structure on Performance of Listed Public Sector Banks in India: International Journal of Business 
and Management Invention, ISSN (online)2319-8028, www. Ijbm.org, Vol. 2, Issue 10/ Oct 2013/ Page 3543

Maryam Masnoon, Abihe Saeed: Cpital

Structure Determinants of KSE Listed Automobile Companies- European Scientific Journal May2014 edition, Vol 10, No 13, ISSN 1857.

Bidyut Jyoti Bhattacharjee, J-S 2010:

Determinants of capital structure of Indian Industries, The Indian journal of commerce, Vol, 63, No. 3.

Goy B. Hatfield, Louis T.W. Cheng and Wallace

N. Davidson-1994: The Determination of
Optimal Capital Structure: The Effect of Firm and Industry Debt Rations on Market Value, Journal of Financial and Strategic Decision, Vol. 07, No.3

Nicos Michaelas, francies Chittenden, PanikkosPoutziouris, (1998) "A Model of Capital Structure Decision Making in Small Firms": Journal of Small Business and Enterprise Development, Vol. 5 lss: 3, pp. 246-260.

Stewart c Myer, Journal of economic perspectives- Vol. 15, No. 2- spring-2001, Myer, page 81-102.

\section{Appendix}

Ultratech Cement

\begin{tabular}{|l|l|l|l|l|l|l|l|}
\hline Year & $\begin{array}{l}\text { X (Owners' } \\
\text { equity }\end{array}$ & $\begin{array}{l}\text { Y(Net } \\
\text { profit) }\end{array}$ & Dx & Dy & $\mathbf{d x}^{2}$ & $\mathbf{d y}^{2}$ & dxdy \\
\hline $2011-12$ & 2174.65 & 20736.1 & -112.5 & 3778.91 & 12655.8 & $1.4 \mathrm{E}+07$ & -425119 \\
\hline $2012-13$ & 2014.74 & 18857.7 & -272.41 & 1900.5 & 74206.1 & 3611885 & -517710 \\
\hline $2013-14$ & 2144.73 & 17097.5 & -142.42 & 140.326 & 20282.9 & 19691.4 & -19985 \\
\hline $2014-15$ & 2655.43 & 15234.8 & 368.282 & -1722.4 & 135632 & 2966538 & -634316 \\
\hline $2015-16$ & 2446.19 & 12859.8 & 159.042 & -4097.4 & 25294.4 & $1.7 E+07$ & -651653 \\
\hline & $\mathbf{1 1 4 3 5 . 7}$ & $\mathbf{8 4 7 8 5 . 9}$ & & & $\mathbf{2 6 8 0 7 1}$ & $\mathbf{3 . 8 E}+\mathbf{0 7}$ & $\mathbf{- 2 E + 0 6}$ \\
\hline Mean & $\mathbf{2 2 8 7 . 1 4 8}$ & $\mathbf{1 6 9 5 7 . 2}$ & & & & & \\
\hline r & $-\mathbf{0 . 7 0 7 6 9}$ & & & & & & \\
\hline
\end{tabular}

Shree Cement

\begin{tabular}{|l|l|l|l|l|l|l|l|}
\hline Year & $\begin{array}{l}\mathbf{X} \text { (Owners' } \\
\text { equity }\end{array}$ & $\begin{array}{l}\text { Y(Net } \\
\text { profit) }\end{array}$ & $\mathbf{D x}$ & $\mathbf{D y}$ & $\mathbf{d x ^ { 2 }}$ & $\mathbf{d y}^{2}$ & $\mathbf{d x d y}$ \\
\hline $2011-12$ & 454.54 & 6180.22 & -203.58 & 1122.71 & 41443.2 & 1260482 & -228557 \\
\hline $2012-13$ & 426.33 & 5276.4 & -231.79 & 218.892 & 53724.8 & 47913.71 & -50736.1 \\
\hline $2013-14$ & 787.24 & 5276.4 & 129.124 & 218.892 & 16673 & 47913.71 & 28264.2 \\
\hline $2014-15$ & 1003.97 & 4710.87 & 345.854 & -346.64 & 119615 & 120157.9 & -119886 \\
\hline $2015-16$ & 618.5 & 3843.65 & -39.616 & -1213.9 & 1569.43 & 1473451 & 48088.2 \\
\hline & $\mathbf{3 2 9 0 . 5 8}$ & $\mathbf{2 5 2 8 7 . 5 4}$ & & & $\mathbf{2 3 3 0 2 5}$ & $\mathbf{2 9 4 9 9 1 9}$ & $\mathbf{- 3 2 2 8 2 7}$ \\
\hline Mean & $\mathbf{6 5 8 . 1 1 6}$ & $\mathbf{5 0 5 7 . 5 0 8}$ & & & & & \\
\hline $\mathbf{r}$ & $\mathbf{0 . 3 8 9 3 7}$ & & & & & & \\
\hline
\end{tabular}


Ambuja Cement

\begin{tabular}{|l|l|l|l|l|l|l|l|}
\hline Year & $\begin{array}{l}\text { X(Owners' } \\
\text { equity }\end{array}$ & $\begin{array}{l}\text { Y(Net } \\
\text { profit) }\end{array}$ & Dx & Dy & $\mathbf{d x}^{2}$ & dy $^{2}$ & dxdy \\
\hline $2011-12$ & 807.56 & 10306.87 & -417.32 & 952.89 & 174158 & 907999 & -397662 \\
\hline $2012-13$ & 1496.36 & 10103.33 & 271.478 & 749.35 & 73700.3 & 561525 & 203432 \\
\hline $2013-14$ & 1294.57 & 9485.54 & 69.688 & 131.56 & 4856.42 & 17308 & 9168.15 \\
\hline $2014-15$ & 1297.06 & 8805.06 & 72.178 & -548.92 & 5209.66 & 301313 & -39620 \\
\hline $2015-16$ & 1228.86 & 8069.1 & 3.978 & -1284.9 & 15.8245 & 1650917 & -5111.3 \\
\hline & $\mathbf{6 1 2 4 . 4 1}$ & $\mathbf{4 6 7 6 9 . 9}$ & & & $\mathbf{2 5 7 9 4 0}$ & $\mathbf{3 4 3 9 0 6 3}$ & $\mathbf{- 2 2 9 7 9 3}$ \\
\hline Mean & $\mathbf{1 2 2 4 . 8 8 2}$ & $\mathbf{9 3 5 3 . 9 8}$ & & & & & \\
\hline r & $\mathbf{- 0 . 2 4 3 9 8}$ & & & & & & \\
\hline
\end{tabular}

ACC Cement

\begin{tabular}{|l|l|l|l|l|l|l|l|}
\hline Year & $\begin{array}{l}\text { X(Owners' } \\
\text { equity }\end{array}$ & $\begin{array}{l}\text { Y(Net } \\
\text { profit) }\end{array}$ & Dx & Dy & $\mathbf{d x}^{\mathbf{2}}$ & $\mathbf{d y}^{\mathbf{2}}$ & $\mathbf{d x d y}$ \\
\hline $\mathbf{2 0 1 1 - 1 2}$ & $\mathbf{7 4 4 . 7 4}$ & 8443.04 & -401.38 & 627.328 & 161109 & 393540 & -251799 \\
\hline $\mathbf{2 0 1 2 - 1 3}$ & 1168.29 & 8235.61 & 22.166 & 419.898 & 491.332 & 176314 & 9307.46 \\
\hline $\mathbf{2 0 1 3 - 1 4}$ & 1095.76 & 7824.84 & -50.364 & 9.128 & 2536.53 & 83.3204 & -459.72 \\
\hline $\mathbf{2 0 1 4 - 1 5}$ & 1396.57 & 7382.8 & 250.446 & -432.91 & 62723.2 & 187413 & -108421 \\
\hline $\mathbf{2 0 1 5 - 1 6}$ & 1325.26 & 7192.27 & 179.136 & -623.44 & 32089.7 & 388680 & -111681 \\
\hline & $\mathbf{5 7 3 0 . 6 2}$ & $\mathbf{3 9 0 7 8 . 6}$ & & & $\mathbf{2 5 8 9 5 0}$ & $\mathbf{1 1 4 6 0 3 1}$ & $\mathbf{- 4 6 3 0 5 4}$ \\
\hline Mean & $\mathbf{1 1 4 6 . 1 2 4}$ & $\mathbf{7 8 1 5 . 7 1}$ & & & & & \\
\hline $\mathbf{r}$ & $\mathbf{- 0 . 8 5 0 0 1}$ & & & & & & \\
\hline
\end{tabular}

Ramco Cement

\begin{tabular}{|l|l|l|l|l|l|l|l|}
\hline Year & $\begin{array}{l}\text { X(Owners' } \\
\text { equity }\end{array}$ & $\begin{array}{l}\text { Y(Net } \\
\text { profit) }\end{array}$ & Dx & Dy & $\mathbf{d x}^{\mathbf{2}}$ & $\mathbf{d y}^{2}$ & Dxdy \\
\hline $\mathbf{2 0 1 1 - 1 2}$ & 558.26 & 3092.63 & 212.846 & 564.422 & 45303.4 & 318572 & 120135 \\
\hline $\mathbf{2 0 1 2 - 1 3}$ & 242.35 & 2645.19 & -103.06 & 116.982 & 10622.2 & 13684.8 & -12057 \\
\hline $\mathbf{2 0 1 3 - 1 4}$ & 137.7 & 2482.08 & -207.71 & -46.128 & 43145.1 & 2127.79 & 9581.43 \\
\hline $\mathbf{2 0 1 4 - 1 5}$ & 403.65 & 2370.76 & 58.236 & -157.45 & 3391.43 & 24789.9 & -9169.1 \\
\hline $\mathbf{2 0 1 5 - 1 6}$ & 385.11 & 2050.38 & 39.696 & -477.83 & 1575.77 & 228320 & -18968 \\
\hline & $\mathbf{1 7 2 7 . 0 7}$ & $\mathbf{1 2 6 4 1}$ & & & $\mathbf{1 0 4 0 3 8}$ & $\mathbf{5 8 7 4 9 4}$ & $\mathbf{8 9 5 2 2 . 8}$ \\
\hline Mean & $\mathbf{3 4 5 . 4 1 4}$ & $\mathbf{2 5 2 8 . 2 1}$ & & & & & \\
\hline r & $\mathbf{- 0 . 3 6 2 1 0 6}$ & & & & & & \\
\hline
\end{tabular}


Prism Cement

\begin{tabular}{|l|l|l|l|l|l|l|l|}
\hline Year & $\begin{array}{l}\text { X(Owners' } \\
\text { equity }\end{array}$ & $\begin{array}{l}\text { Y(Net } \\
\text { profit) }\end{array}$ & Dx & Dy & dx2 & dy $^{2}$ & dxdy \\
\hline $\mathbf{2 0 1 1 - 1 2}$ & 8.36 & 988.45 & 37.976 & -61.582 & 1442.18 & 3792.34 & -2338.6 \\
\hline $\mathbf{2 0 1 2 - 1 3}$ & 14.7 & 1015.37 & 44.316 & -34.662 & 1963.91 & 1201.45 & -1536.1 \\
\hline $\mathbf{2 0 1 3 - 1 4}$ & -81.65 & 1008.06 & -52.034 & -41.972 & 2707.54 & 1761.65 & 2183.97 \\
\hline $\mathbf{2 0 1 4 - 1 5}$ & -59.48 & 1089.71 & -29.864 & 39.678 & 891.859 & 1574.34 & -1184.9 \\
\hline $\mathbf{2 0 1 5 - 1 6}$ & -30.01 & 1148.57 & -0.394 & 98.538 & 0.15524 & 9709.74 & -38.824 \\
\hline & $\mathbf{- 1 4 8 . 0 8}$ & $\mathbf{5 2 5 0 . 1 6}$ & & & $\mathbf{7 0 0 5 . 6 4}$ & $\mathbf{1 8 0 3 9 . 5}$ & $\mathbf{- 2 9 1 4 . 5}$ \\
\hline Mean & $\mathbf{- 2 9 . 6 1 6}$ & $\mathbf{1 0 5 0 . 0 3}$ & & & & & \\
\hline r & $\mathbf{0 . 2 5 9 2 6}$ & & & & & & \\
\hline
\end{tabular}

JK Cement

\begin{tabular}{|l|l|l|l|l|l|l|l|}
\hline Year & $\begin{array}{l}\text { X(Owners' } \\
\text { equity }\end{array}$ & $\begin{array}{l}\text { Y(Net } \\
\text { profit) }\end{array}$ & Dx & Dy & $\mathbf{d x}^{2}$ & $\mathbf{d y}^{2}$ & Dxdy \\
\hline $\mathbf{2 0 1 1 - 1 2}$ & 101.54 & 1714.41 & -53.298 & 136.282 & 2840.68 & 18572.8 & -7263.6 \\
\hline $\mathbf{2 0 1 2 - 1 3}$ & 156.92 & 1646.54 & 2.082 & 68.412 & 4.33472 & 4680.2 & 142.434 \\
\hline $\mathbf{2 0 1 3 - 1 4}$ & 97.03 & 1543.39 & -57.808 & -34.738 & 3341.77 & 1206.73 & 2008.13 \\
\hline $\mathbf{2 0 1 4 - 1 5}$ & 233.55 & 1697.39 & 78.712 & 119.262 & 6195.58 & 14223.4 & 9387.35 \\
\hline $\mathbf{2 0 1 5 - 1 6}$ & 185.15 & 1288.91 & 30.312 & -289.22 & 918.817 & 83647.1 & -8766.8 \\
\hline & $\mathbf{7 7 4 . 1 9}$ & $\mathbf{7 8 9 0 . 6 4}$ & & & $\mathbf{1 3 3 0 1 . 2}$ & $\mathbf{1 2 2 3 3 0}$ & $\mathbf{- 4 4 9 2 . 4}$ \\
\hline Mean & $\mathbf{1 5 4 . 8 3 8}$ & $\mathbf{1 5 7 8 . 1 3}$ & & & & & \\
\hline r & $-\mathbf{0 . 1 1 1 3 7}$ & & & & & & \\
\hline
\end{tabular}

JK Laxmi Cement

\begin{tabular}{|c|c|c|c|c|c|c|c|}
\hline Year & $\begin{array}{l}\text { X(Owners' } \\
\text { equity }\end{array}$ & $\begin{array}{l}Y(\text { Net } \\
\text { profit) }\end{array}$ & Dx & Dy & $d x^{2}$ & $d y^{2}$ & dxdy \\
\hline 2011-12 & 6.28 & 1333.44 & -89.6 & 52.97 & 8028.16 & 2805.82 & -4746.1 \\
\hline 2012-13 & 95.6 & 1330.7 & -0.28 & 50.23 & 0.0784 & 2523.05 & -14.064 \\
\hline 2013-14 & 93 & 1303.22 & -2.88 & 22.75 & 8.2944 & 517.563 & -65.52 \\
\hline 2014-15 & 175.74 & 1259.8 & 79.86 & -20.67 & 6377.62 & 427.249 & -1650.7 \\
\hline \multirow[t]{2}{*}{ 2015-16 } & 108.78 & 1175.19 & 12.9 & -105.28 & 166.41 & 11083.9 & -1358.1 \\
\hline & 479.4 & 6402.35 & & & 14580.6 & 17357.6 & $\begin{array}{l}- \\
7834.5 \\
\end{array}$ \\
\hline Mean & 95.88 & 1280.47 & & & & & \\
\hline$r$ & -0.49247 & & & & & & \\
\hline
\end{tabular}


Orient Cement

\begin{tabular}{|l|l|l|l|l|l|l|l|}
\hline Year & $\begin{array}{l}\text { X(Owners' } \\
\text { equity }\end{array}$ & $\begin{array}{l}\text { Y(Net } \\
\text { profit) }\end{array}$ & Dx & Dy & $\mathbf{d x}^{2}$ & $\mathbf{d y}^{\mathbf{2}}$ & $\mathbf{d x d y}$ \\
\hline $\mathbf{2 0 1 1 - 1 2}$ & 62.24 & 1016.3 & -41.488 & 301.034 & 1721.25 & 90621.5 & -12489 \\
\hline $\mathbf{2 0 1 2 - 1 3}$ & 194.78 & 975.54 & 91.052 & 260.274 & 8290.47 & 67742.6 & 23698.5 \\
\hline $\mathbf{2 0 1 3 - 1 4}$ & 101.02 & 828.76 & -2.708 & 113.494 & 7.33326 & 12880.9 & -307.34 \\
\hline $\mathbf{2 0 1 4 - 1 5}$ & 161.67 & 756.75 & 57.942 & 41.484 & 3357.28 & 1720.92 & 2403.67 \\
\hline $\mathbf{2 0 1 5 - 1 6}$ & -1.07 & -1.02 & -104.8 & -716.29 & 10982.6 & 513066 & 75065.3 \\
\hline & $\mathbf{5 1 8 . 6 4}$ & $\mathbf{3 5 7 6 . 3 3}$ & & & $\mathbf{2 4 3 5 9}$ & $\mathbf{6 8 6 0 3 2}$ & $\mathbf{8 8 3 7 0 . 8}$ \\
\hline Mean & $\mathbf{1 0 3 . 7 2 8}$ & $\mathbf{7 1 5 . 2 6 6}$ & & & & & \\
\hline r & $\mathbf{- 0 . 6 8 3 6 0 9}$ & & & & & & \\
\hline
\end{tabular}

OCL India Cement

\begin{tabular}{|l|l|l|l|l|l|l|l|}
\hline Year & $\begin{array}{l}\text { X(Owners' } \\
\text { equity }\end{array}$ & $\begin{array}{l}\text { Y(Net } \\
\text { profit) }\end{array}$ & Dx & Dy & $\mathbf{d x 2}$ & $\mathbf{d y}^{2}$ & $\mathbf{d x d y}$ \\
\hline $\mathbf{2 0 1 1 - 1 2}$ & 236.29 & 1405.11 & 109.078 & 274.52 & 11898 & 75361.2 & 29944.1 \\
\hline $\mathbf{2 0 1 2}-13$ & 113.69 & 1196.21 & -13.522 & 65.62 & 182.845 & 4305.98 & -887.31 \\
\hline $\mathbf{2 0 1 3 - 1 4}$ & 97.88 & 1109.92 & -29.332 & -20.67 & 860.366 & 427.249 & 606.292 \\
\hline $\mathbf{2 0 1 4 - 1 5}$ & 156.39 & 1038.67 & 29.178 & -91.92 & 851.356 & 8449.29 & -2682 \\
\hline $\mathbf{2 0 1 5 - 1 6}$ & 31.81 & 903.04 & -95.402 & -227.55 & 9101.54 & 51779 & 21708.7 \\
\hline & $\mathbf{6 3 6 . 0 6}$ & $\mathbf{5 6 5 2 . 9 5}$ & & & $\mathbf{2 2 8 9 4 . 1}$ & $\mathbf{1 4 0 3 2 3}$ & $\mathbf{4 8 6 8 9 . 8}$ \\
\hline Mean & $\mathbf{1 2 7 . 2 1 2}$ & $\mathbf{1 1 3 0 . 5 9}$ & & & & & \\
\hline r & $\mathbf{0 . 8 5 9 0 3 6}$ & & & & & & \\
\hline
\end{tabular}

